\title{
An Evaluation of Successive Pilot Decontamination in Massive MIMO
}

\section{Uma Avaliação da Descontaminação Piloto Sucessiva em MIMO de Larga Escala}

\author{
Victor Croisfelt Rodrigues ${ }^{1}$; Taufik Abrão ${ }^{2}$
}

\begin{abstract}
The demand for higher data rates can be satisfied by the spectral efficiency (SE) improvement offered by Massive multiple-input multiple-output (M-MIMO) systems. However, the pilot contamination remains as a fundamental issue to obtain the paramount SE in such systems. This has been propitiating the research of several methods to mitigate pilot contamination. One of these procedures is based on the coordination of the cells, culminating in proposals with multiple pilot training phases. This paper aims to expand the results of the original paper, whereby the concepts of large pilot training phases were offered. The evaluation of such method was conducted through more comprehensible numerical results, in which a large number of antennas were assumed and more rigorous SE expressions were used. The channel estimation approaches relying on multiple pilot training phases were considered cumbersome for implementation and an uninteresting solution to overcome pilot contamination; contradicting the results presented in the genuine paper.
\end{abstract}

Keywords: Massive MIMO. Pilot Contamination. Mitigate. Coordination of Cells.

\section{Resumo}

A demanda por maiores taxas de dados pode ser satisfeita pela melhoria da eficiência espectral fornecida pelos sistemas multiple-input multiple-output de larga escala. No entanto, a contaminação piloto continua a ser um problema fundamental para obter a eficiência espectral primordial em tais sistemas. Isto propiciou a pesquisa de diversos métodos para mitigar a contaminação piloto. Um desses procedimentos é baseado na coordenação das células, culminando em propostas com múltiplas fases de treinamento piloto. Este artigo tem como objetivo ampliar os resultados do trabalho original, através do qual os conceitos de grandes fases de treinamento piloto foram oferecidos. A avaliação de tal método foi realizada por meio de resultados númericos mais compreensíveis, em que um grande número de antenas foi assumido e expressões mais rigorosas de eficiência espectral foram usadas. As abordagens de estimativa de canal baseadas em múltiplas fases de treinamento piloto foram consideradas problemáticas para implementação e uma solução desinteressante para superar a contaminação piloto; contradizendo os resultados apresentados no artigo original.

Palavras-chave: MIMO de Larga Escala. Contaminação Piloto. Mitigação. Coordenação das Células.

\footnotetext{
${ }^{1}$ Discente, Bacharelado, Engenharia Elétrica, UEL, Londrina, PR, Brasil; E-mail: victorcroisfelt@gmail.com

2 Dr. Prof. Depto. de Engenharia Elétrica, UEL, Londrina, PR, Brasil, E-mail: taufik@uel.br
} 


\section{Introduction}

M-MIMO technology has been extensively studied with the intention of being deployed in the fifth generation (5G) of cellular systems. This comes mainly because of its ability to improve the SE and to considerably reduce the wireless harmful effects. In order to enable the MMIMO operation, the base stations (BSs) should estimate the wireless channel between them and their respective numerous user equipment (UE) (MARZETTA et al., 2016). To this end, the estimation of channel vectors is obtained through the so called pilot training phase. In this period, the UEs transmit pilot sequences to their respective BS in a time-division duplex (TDD) mode, wherein these pilots are recognized at both link ends. However, note that the number of UEs is growing large in the current scenarios of interest, and the high mobility circumstances are becoming more usual, e.g. receiving and transmitting data through Internet while traveling in a car. Then, the pilot sequences are often reused across the BSs, as a consequence the channel estimates are contaminated by the non-orthogonality of these pilots. This issue is commonly known as pilot contamination.

Pilot contamination mitigation techniques ${ }^{1}$ are fundamental to improve the performance of M-MIMO systems as reported in ELIJAH et al. (2016). Consequently, approaches to reduce the pilot contamination effect have been the focus of several works. Herein, we are interested in discuss a particular manner proposed by VU; VU; QUEK (2014), which relies on the coordination between cells. The authors of this latter paper proposed a mitigation scheme comprised of multiple pilot training phases, aiming to explore the additional information that arose thereof to eliminate successively the interference between the cells. Besides, this procedure was credited as a favorable solution to the pilot contamination problem.

That being said, this paper aims to extent the results obtained in VU; VU; QUEK (2014) to a more suitable M-MIMO condition ${ }^{2}$ and also calls attention to the drawbacks provided by the application of this technique. With this in mind, we derived the equations of the two proposed procedures for channel estimation presented in $\mathrm{VU}$; VU; QUEK (2014), which exploit the multiple pilot training information. In order to get a more tight lower bound expression for the SE when applying a maximum-ratio (MR) combiner, the mathematical framework treated here is ob-

\footnotetext{
The mitigation of pilot contamination is also known as pilot decontamination.

2 In VU; VU; QUEK (2014), the maximum number of BS antennas used was thirty.
}

tained assuming a minimum mean-squared error (MMSE) channel estimation procedure. In this paper, we first discuss a canonical multi-cell M-MIMO system, introducing either the pilot training and the uplink (UL) payload data phases. The successive pilot decontamination approach proposed by VU; VU; QUEK (2014) is then examined. Lastly, illustrative numerical results are presented and final remarks are offered.

Notations: The superscript ${ }^{\mathrm{H}}$ denotes the Hermitian transpose. The $\mathscr{N}_{\mathbb{C}}\left(\mu, \sigma^{2}\right)$ stands for the circularly symmetric complex Gaussian distribution with a mean $\mu$ and standard deviation $\sigma$. The statistical operator $\mathbb{E}\{x\}$ represents the expected value of a random variable $x$. The $N \times N$ identity matrix is indicated by $\mathbf{I}_{N}$.

\section{System Model}

A canonical M-MIMO system comprised of $L$ cells operating synchronously and sharing the same timefrequency resources is presented in this section. Each cell has a BS located at the center of the cell and equipped with an array of $M$ antenna elements, whereby $K$ singleantenna UEs are served. The synchronous activities and equal number of UEs within each cell are assumed only to simplify the upcoming formulation and both ones could be relaxed. Throughout this work, our interest is on the operation of a specific cell called as the home cell and denoted by the index $j$.

The cells are situated in a fully isotropic fading environment wherein the link between a UE and the antenna array at $\mathrm{BS} j$ is considered to be static within a $\tau_{\mathrm{c}}$-length block, that is a coherence interval ${ }^{3}$. It is also said that the realization of each block is statistically independent. Then, the $M \times 1$ vector representing the wireless channel from UE $k$ within cell $l$ to BS $j$ is denoted as: $\mathbf{g}_{j l k}=\sqrt{\beta_{j l k}} \mathbf{h}_{j l k}$, where $\mathbf{h}_{j l k} \in \mathbb{C}^{M \times 1}$ represents the small-scale fading with a Rayleigh distribution, $\mathbf{h}_{j l k} \sim \mathscr{N}_{\mathbb{C}}\left(\mathbf{0}, \mathbf{I}_{M}\right)$. Moreover, $\beta_{j l k}$ is the average large-scale fading coefficient, which incorporates the macroscopic propagation phenomena that change slowly over time. Note that $\beta_{j l k}$ is only related to the UEs, being considered identical for all elements of the antenna array. We can rewrite the channel vector in a purely statistical form, as $\mathbf{g}_{j l k} \sim \mathscr{N}_{\mathbb{C}}\left(\mathbf{0}, \beta_{j l k} \mathbf{I}_{M}\right)$, where $\beta_{j l k} \mathbf{I}_{M}$ is the channel covariance matrix. Henceforth, we will assume that the average large-scale coefficients are known a priori for anyone who needs to know them. In

\footnotetext{
Recall that a coherence interval is a time-frequency period in which the channel is said to be approximately static and is given as $\tau_{\mathrm{c}}=B_{\mathrm{c}} T_{\mathrm{c}}$, where $T_{\mathrm{c}}$ is the coherence time and $B_{\mathrm{c}}$ is the coherence bandwidth (MARZETTA et al., 2016)
} 
fact, this last consideration comprises an idealistic scenario, once an estimation phase is required to obtain the values of $\beta_{j l k}$; however, this discussion is beyond the scope of this work.

\section{Uplink Pilots and Channel Estimation}

The pilot training phase consists of all UEs in the system transmitting their $\tau_{\mathrm{p}}$-length pilot sequences to their own BS. In our context, the pilots are reused among the $L$ cells, merely as a necessity, as a result of the difficult to generate a large number of orthogonal pilot sequences inside a short coherence interval. For ease of exposition, we consider that $\tau_{\mathrm{p}}=K$, where the $i$ th UE of each cell is assigned with the same pilot. Otherwise, it is assumed that the pilots are mutually orthogonal. It is therefore possible to note that the intra-cell interference is negligible in the estimation phase, while the inter-cell interference impacts the system performance. Additionally, one can emphasize that those pilots are randomly assigned across the UEs in each cell, indicating the usage of a classical assignment scheme, as made in MARZETTA et al. (2016).

The received pilot signal in the home cell is a linear combination of the transmitted pilots from all cells, yielding in the following received pilot signal matrix:

$$
\mathbf{Y}_{j}^{\mathrm{p}}=\sqrt{\rho^{\mathrm{p}}} \sum_{l=1}^{L} \sum_{k=1}^{K} \mathbf{g}_{j l k} \phi_{k}^{\mathrm{H}}+\mathbf{N}_{j}^{\mathrm{p}}
$$

where $\mathbf{Y}_{j}^{\mathrm{p}} \in \mathbb{C}^{M \times \tau_{\mathrm{p}}}$ and $\rho^{\mathrm{p}}$ is the normalized ${ }^{4}$ transmit power spent on pilot training and is given by $\rho^{\mathrm{p}}=\tau_{\mathrm{p}} \rho^{\mathrm{ul}}$, being $\rho^{\text {ul }}$ the UL normalized power. Furthermore, $\phi_{k} \in$ $\mathbb{C}^{\tau_{\mathrm{p}}}$ is the pilot assigned to UE $k$, which has zero mean and unit norm: $\left\|\phi_{k}\right\|^{2}=\phi_{k}^{\mathrm{H}} \phi_{k}=1 . \mathbf{N}_{l}^{\mathrm{p}} \in \mathbb{C}^{M \times \tau_{\mathrm{p}}}$ is the receiver noise matrix with each element modeled as an independent and identically distributed (i.i.d.) additive white Gaussian noise, which follows $\mathscr{N}_{\mathbb{C}}(0,1)$.

Afterwards, the home cell BS correlates the received signal with the pilot sequences allocated to each UE, resulting in $K$ de-spreading signals to each BS. This operation can be defined for the $i$ th UE within cell $j$ as

$$
\mathbf{y}_{j i}^{\mathrm{pd}} \triangleq \mathbf{Y}_{j}^{\mathrm{p}} \phi_{i}=\sqrt{\rho^{\mathrm{p}}} \sum_{l=1}^{L} \mathbf{g}_{j l i}+\mathbf{n}_{j i}^{\mathrm{pd}},
$$

where $\mathbf{n}_{j i}^{\mathrm{pd}} \in \mathbb{C}^{M}$ is an equivalent white Gaussian noise vector with i.i.d. $\mathscr{N}_{\mathbb{C}}(0,1)$ elements. The latter distribution is maintained because of the linearity involving the

$4 \quad$ The term "normalized" signifies that the transmit power is already taking into account the noise power. de-spreading operation. The above de-spreading signal represents the neat observation acquired in the $\mathrm{BS} j$, in the sense that it is not dependent on the pilot sequences. That being the case, estimation techniques can be applied thereon to obtain the channel vectors in a given coherence block. Also, note that the linear combination with the UEs within other cells different from $j$ represents the pilot contamination effect.

The observation in (2) is utilized by BS $j$ to estimate its respective channel vectors. Assuming that we know some prior knowledge of the observation, as its distribution and statistical parameters (mean and variance), a Bayesian approach can be adopted to obtain the channel estimates. Due to the fact that the observation follows a circularly complex Gaussian distribution, the MMSE estimator is explicit to be obtained in order to acquire an estimate of $\mathbf{g}_{j l k}$. This estimation procedure realized in the BS $j$ and based on the observation $\mathbf{y}_{j k}^{\mathrm{pd}}$ is given as (KAY, 1993)

$$
\begin{aligned}
\hat{\mathbf{g}}_{j l k} & =\mathbb{E}\left\{\mathbf{g}_{j l k} \mid \mathbf{y}_{j k}^{\mathrm{pd}}\right\} \\
& =\frac{\sqrt{\rho^{\mathrm{p}}} \beta_{j l k}}{1+\rho^{\mathrm{p}} \sum_{l^{\prime}=1}^{L} \beta_{j l^{\prime} k}} \mathbf{y}_{j k}^{\mathrm{pd}} .
\end{aligned}
$$

According to the orthogonality principle (KAY, 1993), the estimate, $\hat{\mathbf{g}}_{j l k}$, and the estimation error, $\tilde{\mathbf{g}}_{j l k}=\hat{\mathbf{g}}_{j l k}-$ $\mathbf{g}_{j l k}$, are uncorrelated random vectors. This assertion implicates independence for Gaussian distributed quantities. Also, they are statistically given out as $\hat{\mathbf{g}}_{j l k} \sim$ $\mathscr{N}_{\mathbb{C}}\left(\mathbf{0}, \psi_{j l k} \mathbf{I}_{M}\right)$ and $\tilde{\mathbf{g}}_{j l k} \sim \mathscr{N}_{\mathbb{C}}\left(\mathbf{0},\left(\beta_{j l k}-\psi_{j l k}\right) \mathbf{I}_{M}\right)$ with

$$
\psi_{j l k}=\frac{\rho^{\mathrm{p}} \beta_{j l k}^{2}}{1+\rho^{\mathrm{p}} \sum_{l^{\prime}=1}^{L} \beta_{j l^{\prime} k}} .
$$

Note that the channel estimates from UEs that are assigned with the same pilots sequences are correlated as

$$
\mathbb{E}\left\{\hat{\mathbf{g}}_{j l k} \hat{\mathbf{g}}_{j l^{\prime} k}^{\mathrm{H}}\right\}=\frac{\rho^{\mathrm{p}} \beta_{j l k} \beta_{j l^{\prime} k}}{1+\rho^{\mathrm{p}} \sum_{l^{\prime \prime}=1}^{L} \beta_{j l^{\prime \prime} k}} .
$$

Thus, observe that the $\mathrm{BS}$ is unable to separate the contaminating UEs, emphasizing the severe impact caused by pilot contamination.

\section{Uplink Data Transmission}

During UL data transmission, the UEs independently transmit they data streams to their own BS. The received 
baseband signal in $\mathrm{BS} j$ is given as a linear combination of the $K$ transmitted signals over the entire system:

$$
\mathbf{y}_{j}=\sqrt{\rho^{\mathrm{ul}}} \sum_{l=1}^{L} \sum_{k=1}^{K} \mathbf{g}_{j l k} s_{l k}+\mathbf{n}_{j}
$$

where $\mathbf{y}_{j} \in \mathbb{C}^{M}, s_{l k} \sim \mathscr{N}_{\mathbb{C}}(0,1)$ is the information symbol transmitted by UE $k$ within cell $l$ and $\mathbf{n}_{j} \sim \mathscr{N}_{\mathbb{C}}\left(\mathbf{0}, \mathbf{I}_{M}\right)$ is the receiver noise.

The BS is capable to detect the symbol transmitted by a desired UE using a linear decoding technique applied via a receive combining vector; which is denoted as $\mathbf{v}_{j k} \in$ $\mathbb{C}^{M}$ for UE $k$ attached to BS $j$. The detection is simply performed as $\mathbf{v}_{j k}^{\mathrm{H}} \mathbf{y}_{j}$, in which we consider the utilization of the basic maximum-ratio (MR) combiner defined as (HOYDIS; BRINK; DEBBAH, 2013)

$$
\mathbf{v}_{j k}^{\mathrm{H}} \triangleq \frac{1}{\sqrt{M}} \hat{\mathbf{g}}_{j j k}^{\mathrm{H}}
$$

Note that only the channel estimates within cell $j$ are needed to detect the messages of all UEs for MR.

The ergodic UL capacity can be obtained by applying a use-and-forget technique, as proposed in MARZETTA et al. (2016). Using this procedure, the BS is considered to neglect its knowledge of the channel estimates, allowing the derivation of a tight lower bound expression. The instantaneous SE for UE $k$ within cell $j$ is then lower bounded by (MARZETTA et al., 2016)

$$
\mathrm{SE}_{j k}^{\mathrm{ul}}=\left(1-\frac{\tau_{\mathrm{p}}}{\tau_{\mathrm{c}}}\right) \mathbb{E}\left\{\log _{2}\left(1+\gamma_{j k}^{\mathrm{ul}}\right)\right\}
$$

where the expectation is relative to the second order statistic realizations and the pre-log factor quantifies the energy used on UL payload transmission. To compute the SE in (8), it is necessary to obtain the effective signal-tointerference-and-noise ratio (SINR), $\gamma_{j k}^{\mathrm{ul}}$, which is determined by the power computation of the combining process $\mathbf{v}_{j k}^{\mathrm{H}} \mathbf{y}_{j}$. Thus, the effective SINR for the MR combiner is (MARZETTA et al., 2016)

$$
\gamma_{j k}^{\mathrm{mr}, \mathrm{ul}}=\frac{M \rho^{\mathrm{ul}} \psi_{j j k}}{1+\rho^{\mathrm{ul}} \sum_{l=1}^{L} \sum_{k=1}^{K} \beta_{j l k}+M \rho^{\mathrm{ul}} \sum_{l=1, l \neq j}^{L} \psi_{j l k}}
$$

\section{Successive Elimination of Pilot Contamination}

The effects of inter-cell pilot contamination in a multicell M-MIMO system were entirely eliminated in the estimation methods proposed by VU; VU; QUEK (2014). The strategy adopted in this cited work relies on the coordination of cells to realize multiple pilot training phases. The knowledge provided by these periods is then applied to successively mitigate the pilot contamination interference.

The multiple phases stem from the realization of pilot transmissions that occur within $L+1$ phases; each with a length of $\tau_{\mathrm{p}}$ samples. In the first phase, denoted as $s=$ 0 , all UEs synchronously transmit the assigned pilots to their respective BS. In the next $L$ phases, indicating the $l$ th phase as $s=l$, the cell $l$ stays silent while the UEs within the other $L-1$ cells are transmitting the same pilot sequence assigned to them in the first phase. Therefore, the multiple pilot training phases last $(L+1) \tau_{\mathrm{p}}$ symbols, i.e., $L$ times more than the classical scheme. Under the aforementioned circumstances, the $\mathrm{BS} j$ receives in the sth pilot training phase:

$$
\mathbf{Y}_{j}^{\mathrm{p}}(s)=\sqrt{\rho^{\mathrm{p}}} \sum_{\substack{l=1 \\ l \neq s}}^{L} \sum_{\substack{k=1 \\ \mathbf{g}_{j l k}}} \boldsymbol{\phi}_{k}^{\mathrm{H}}+\mathbf{N}_{j}^{\mathrm{p}}(s)
$$

where $s=\{0,1, \ldots, L\}$. One observes that the received signal and the white Gaussian noise are dependent of the phase. As a first lack of this scheme, it is possible to note that the extension of the pilot period causes the reduction in the quantity of UEs served within a coherence period regarding the canonical manner. The issue from now on is to evaluate if such lack reflects in some SE gain.

By using (10), the authors in VU; VU; QUEK (2014) proposed two channel estimation schemes to cease with pilot contamination. In contrast to the presented in the last cited work, here we will apply the MMSE channel estimation instead of the least-squares (LS), aiming to exploit the closed-form expressions of the SE provided by the former.

\section{Estimator 1}

The first estimator is based on exploring the first phase and the $j$ th phase of the multiple pilot training phases. Notice that when the BS $j$ remains silent, it collects the interference information from the neighbor cells. So, subtracting the phase 0 from $j$, we have (VU; VU; QUEK, 2014)

$$
\begin{aligned}
\tilde{\mathbf{Y}}_{j, 1}^{\mathrm{p}} & =\mathbf{Y}_{j}^{\mathrm{p}}(0)-\mathbf{Y}_{j}^{\mathrm{p}}(j) \\
& =\sqrt{\rho^{\mathrm{p}}} \sum_{k=1}^{K} \mathbf{g}_{j j k} \phi_{k}^{\mathrm{H}}+\tilde{\mathbf{N}}_{j, 1}^{\mathrm{p}},
\end{aligned}
$$


where $\tilde{\mathbf{N}}_{j, 1}^{\mathrm{p}}=\mathbf{N}_{j}^{\mathrm{p}}(0)-\mathbf{N}_{j}^{\mathrm{p}}(j)$. Realize that $\mathbf{N}_{j}^{\mathrm{p}}(0)$ and $\mathbf{N}_{j}^{\mathrm{p}}(j)$ are statistically independent and then, the entries of $\tilde{\mathbf{N}}_{j, 1}^{\mathrm{p}}$ are i.i.d. $\mathscr{N}_{\mathbb{C}}(0,2)$ distributed. For the $i$ th UE within cell $j$, the de-spreading signal of (11) with $\phi_{k}$ is

$$
\tilde{\mathbf{y}}_{j, 1}^{\mathrm{pd}}=\tilde{\mathbf{Y}}_{j, 1}^{\mathrm{p}} \phi_{k}=\sqrt{\rho^{\mathrm{p}}} \mathbf{g}_{j j k}+\tilde{\mathbf{n}}_{j, 1}^{\mathrm{pd}} .
$$

Considering the observation in (12), the MMSE estimation of $\mathbf{g}_{j j k}$ is straightforwardly defined as (KAY, 1993)

$$
\hat{\mathbf{g}}_{j j k}^{\mathrm{er} 1}=\mathbb{E}\left\{\mathbf{g}_{j j k} \mid \tilde{\mathbf{y}}_{j, 1}^{\mathrm{pd}}\right\}=\frac{\sqrt{\rho^{\mathrm{p}}} \beta_{j j k}}{2+\rho^{\mathrm{p}} \beta_{j j k}} \tilde{\mathbf{y}}_{j, 1}^{\mathrm{pd}} .
$$

The estimate $\hat{\mathbf{g}}_{j j k}^{\mathrm{er}}$ and the estimation error $\tilde{\mathbf{g}}_{j j k}^{\mathrm{er}}$ are again independent and distributed as follows: $\hat{\mathbf{g}}_{j j k}^{\mathrm{er1}} \sim$ $\mathscr{N}_{\mathbb{C}}\left(\mathbf{0}, \psi_{j j k}^{\mathrm{er} 1} \mathbf{I}_{M}\right)$ and $\tilde{\mathbf{g}}_{j j k}^{\mathrm{er} 1} \sim \mathscr{N}_{\mathbb{C}}\left(\mathbf{0},\left(\beta_{j j k}-\psi_{j j k}^{\mathrm{er} 1}\right) \mathbf{I}_{M}\right)$ with

$$
\psi_{j j k}^{\mathrm{er} 1}=\frac{\rho^{\mathrm{p}} \beta_{j j k}^{2}}{2+\rho^{\mathrm{p}} \beta_{j j k}} .
$$

In this way, the channel vectors attached to $\mathrm{BS} j$ are estimated as soon as the BS receives the interference from other cells.

\section{Estimator 2}

Unlike the first estimator, the second one stands to explore the knowledge provided by all $L+1$ phases. This signifies a more accurate information of the interference, consequently a better estimation performance can be acquired The concept lies in take the sum of all sth phases with $s \neq 0$ and $s \neq j$. This summation contains $L-1$ times the signal of interest denoted by the $k$ th UE inside cell $j$ and $L-2$ times the sum of all interferences. The offered operation leads to (VU; VU; QUEK, 2014)

$$
\begin{aligned}
\tilde{\mathbf{Y}}_{j, 2}^{\mathrm{p}} & =\mathbf{Y}_{j}^{\mathrm{p}}(0)+\sum_{\substack{s=1 \\
s \neq j}}^{L} \mathbf{Y}_{j}^{\mathrm{p}}(s)-(L-1) \mathbf{Y}_{j}^{\mathrm{p}}(j) \\
& =L \sqrt{\rho^{\mathrm{p}}} \sum_{k=1}^{K} \mathbf{g}_{j j k} \phi_{k}^{\mathrm{H}}+\tilde{\mathbf{N}}_{j, 2}^{\mathrm{p}},
\end{aligned}
$$

where $\tilde{\mathbf{N}}_{j, 2}^{\mathrm{p}}=\mathbf{N}_{j}^{\mathrm{p}}(0)+\sum_{s=1, s \neq j}^{L} \mathbf{N}_{j}^{\mathrm{p}}(s)-(L-1) \mathbf{N}_{j}^{\mathrm{p}}(l)$. Recalling the independence of the noise components at each phase, the elements of $\tilde{\mathbf{N}}_{j, 2}^{\mathrm{p}}$ are i.i.d. $\mathscr{N}_{\mathbb{C}}\left(0, L^{2}-L+1\right)$.
The de-spreading signal for a UE $k$ inside cell $j$ is given by

$$
\begin{aligned}
\tilde{\mathbf{y}}_{j, 2}^{\mathrm{pd}} & =\tilde{\mathbf{Y}}_{j, 2}^{\mathrm{p}} \phi_{k} \\
& =L \sqrt{\rho^{\mathrm{p}}} \mathbf{g}_{j j k}+\tilde{\mathbf{n}}_{j, 2}^{\mathrm{pd}} .
\end{aligned}
$$

Again, the MMSE channel estimation is promptly defined as

$$
\begin{aligned}
\hat{\mathbf{g}}_{j j k}^{\mathrm{es} 2} & =\mathbb{E}\left\{\mathbf{g}_{j j k} \mid \tilde{\mathbf{y}}_{j, 2}^{\mathrm{pd}}\right\} \\
& =\frac{L \sqrt{\rho^{\mathrm{p}}} \beta_{j j k}}{\left(L^{2}-L+1\right)+L^{2} \rho^{\mathrm{p}} \beta_{j j k}} \tilde{\mathbf{y}}_{j, 2}^{\mathrm{pd}},
\end{aligned}
$$

where $\hat{\mathbf{g}}_{j j k}^{\mathrm{es} 2} \sim \mathscr{N}_{\mathbb{C}}\left(\mathbf{0}, \psi_{j j k}^{\mathrm{es} 2} \mathbf{I}_{M}\right)$ and $\tilde{\mathbf{g}}_{j j k}^{\mathrm{es} 2} \sim \mathscr{N}_{\mathbb{C}}\left(\mathbf{0},\left(\beta_{j j k}-\right.\right.$ $\left.\left.\psi_{j j k}^{\mathrm{es} 2}\right) \mathbf{I}_{M}\right)$, being these uncorrelated. The average covariance of the estimate is given as

$$
\psi_{j j k}^{\mathrm{es} 2}=\frac{L^{2} \rho^{\mathrm{p}} \beta_{j j k}^{2}}{\left(L^{2}-L+1\right)+L^{2} \rho^{\mathrm{p}} \beta_{j j k}} .
$$

\section{Numerical Results}

The performance of an M-MIMO system is investigated along this section, by the evaluation of the different channel estimation procedures presented. Inspired by MARZETTA et al. (2016), it is considered a dense urban multi-cell scenario, which is comprised of seven hexagon cells; each with a radius of $500 \mathrm{~m}$. The home cell is the center cell of this arrangement. The single-antenna UEs are uniformly distributed within the area of the cells, where their average large-scale coefficients are simply modeled as

$$
\beta_{j l k}=\frac{\xi_{j l k}}{d_{j l k}^{\kappa}},
$$

where $\xi_{j l k}$ is the log-normally distributed shadow-fading, i.e., $10 \log _{10}\left(\xi_{j l k}\right) \sim \mathscr{N}\left(0, \sigma_{\text {shad. }}^{2}\right)$, being $\sigma_{\text {shad. }}$ its standard deviation. $d_{j l k}$ is the distance from UE $k$ within cell $l$ to $\mathrm{BS} j$ and $\kappa$ is the decay or pathloss exponent. In our simulations, we adopted $\sigma_{\text {shad. }}=8 \mathrm{~dB}$ and $\kappa=3.76$, whereas other parameters are described in table 1 (MARZETTA et al., 2016). Recall that these values are motivated by the adoption of a dense-urban scenario under a non-line of sight circumstance.

Since classical and successive schemes have different durations, it is desirable to specify a fair scenario to 
Table 1: Simulation parameters for a dense urban scenario.

\begin{tabular}{ll}
\hline Parameter & Value \\
\hline Carrier frequency & $1.9 \mathrm{GHz}$ \\
Spectral bandwidth & $20 \mathrm{MHz}$ \\
Coherence bandwidth $\left(B_{\mathrm{c}}\right)$ & $200 \mathrm{kHz}$ \\
Coherence time $\left(T_{\mathrm{c}}\right)$ & $1 \mathrm{~ms}$ \\
Coherence interval $\left(\tau_{\mathrm{c}}\right)$ & $200 \mathrm{samples}$ \\
Pilot length $\left(\tau_{\mathrm{p}}\right)$ & $K$ \\
Noise variance & $-90.99 \mathrm{dBm}$ \\
Radiated power per terminal & $200 \mathrm{~mW}$ \\
Monte Carlo Realizations & 1000 \\
\hline
\end{tabular}

Source: The authors

make them comparable. With this intention, the classical approach is set to serve a number of $K_{\text {class. }}=70$ UEs, whereas the successive schemes attend to $K_{\text {succ. }}=10$ UEs; in such a way $K_{\text {class. }}=L K_{\text {succ. }}$. This causes the both schemes last the same time interval in the pilot training phase. Moreover, this condition is being used throughout this section.

\section{Performance Evaluation of Channel Estimation}

The channel estimation performance of the presented MMSE estimators in (3), (13) and (17) is evaluated by means of a metric defined from the Bayesian approach of the mean-squared error (MSE) (KAY, 1993). In particular, it is adopted the averaged normalized mean-square error (NMSE) defined as:

$$
\mathrm{NMSE}_{j}^{i}=10 \log _{10}\left(\frac{1}{K} \sum_{k=1}^{K} \frac{\beta_{j j k}-\psi_{j j k}^{i}}{\beta_{j j k}}\right), i=0,1,2 .
$$

where $i=0$ indicates the classical scheme, $i=1$ the first estimator provided by the multiple pilot training phases and $i=2$ the second estimator also regarding this last proposal. The above metric expresses the relative amount of error obtained over the channel estimation phase in $\mathrm{dB}$ per UE per antenna.

Figure 1 shows the average NMSE as a function of the radiated power per UE for the classical and the estimation methods discussed. In addition to these curves, the canonical single-cell M-MIMO system was also plotted, which consists of the sole operation of the home cell. Therefore, this single-cell system is only affected by the receiver noise.

As can be seen, the estimation performance of the successive schemes are better than the multi-cell classical
Figure 1: Average NMSE as a function of the radiated power per UE for $M=100$ antennas.

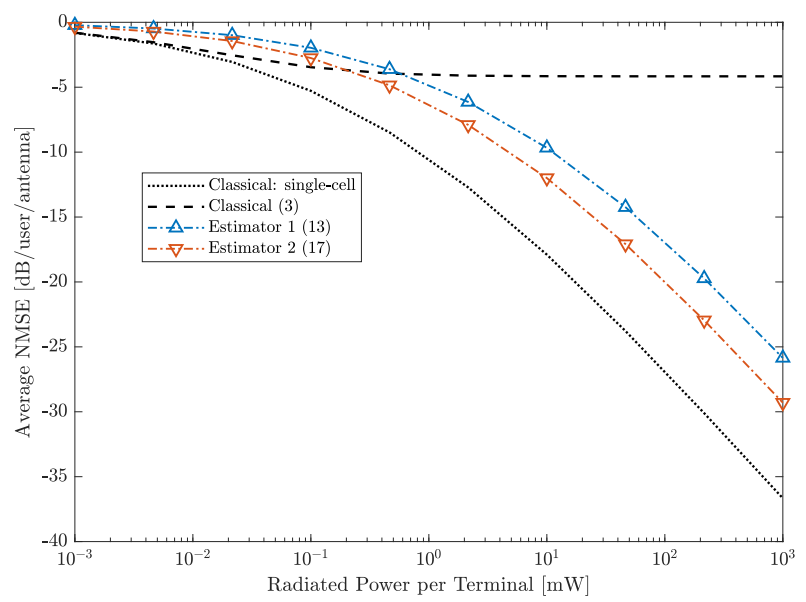

Source: The authors.

strategy from a radiated power of almost $1 \mathrm{~mW}$. Thus, one needs to keep in mind that the former are not always better than the multi-cell case. This occurs due to the knowledge gathered in the multiple pilot training phases has been acquired through the sum of independent noise realizations, as a result the noise variance is increased in the successive schemes. It is therefore possible to infer that the successive estimators may not support a good performance for UEs with the worst channel conditions, bearing in mind that the radiated power per UE is proportional to the signal-to-noise ratio (SNR). In fact, this result was not discussed and demonstrated in VU; VU; QUEK (2014).

Also, note that the successive channel estimations have a similar behavior with respect to the single-cell curve. As a matter of fact, the estimator for single-cell only differs in the noise variance from the techniques exhibited in (13) and (17); once its noise variance is trivially equal to 1 . Lastly, notice that the estimator 1 provides a worse quality of channel estimation than the acquired by estimator 2 .

\section{Spectral Efficiency Analysis}

The average UL SE and the average sum UL SE are illustrated respectively in Figure 2 and Figure 3 for several values of $M$. The authors in VU; VU; QUEK (2014) pointed out that their estimators have a greater SE per UE, in view of the better channel estimates obtained by the successive schemes. This fact was confirmed in our simulation results, where the gains provided by the successive approaches are in average $6.93 \%$ for estimator 1 and $6.98 \%$ for estimator 2 regarding the classical multi-cell case. These values also permit to say that the dif- 
Figure 2: Average UL SE versus $M$ with the radiated power set to $200 \mathrm{~mW}$. The single-cell case indicates the classical scheme in which only the home cell is operating.

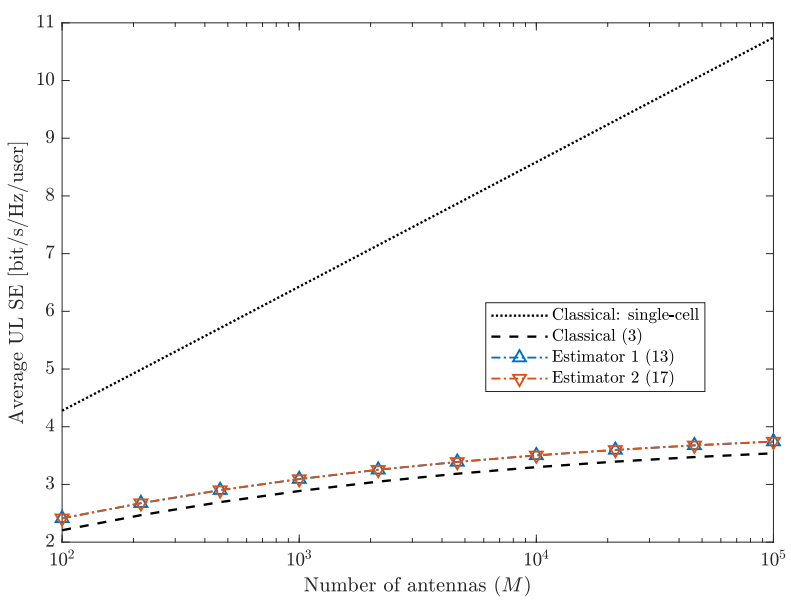

Source: The authors.

ference between the successive estimators are not so great. Since the BSs obtain their respective channel estimates quicker and less data is stored over the procedure, the estimator 1 is preferable and does not imply a considerable loss in SE per UE with respect to estimator 2.

Figure 3: Average UL sum SE versus $M$ with the radiated power set to $200 \mathrm{~mW}$. The single-cell case indicates the classical scheme in which only the home cell is operating.

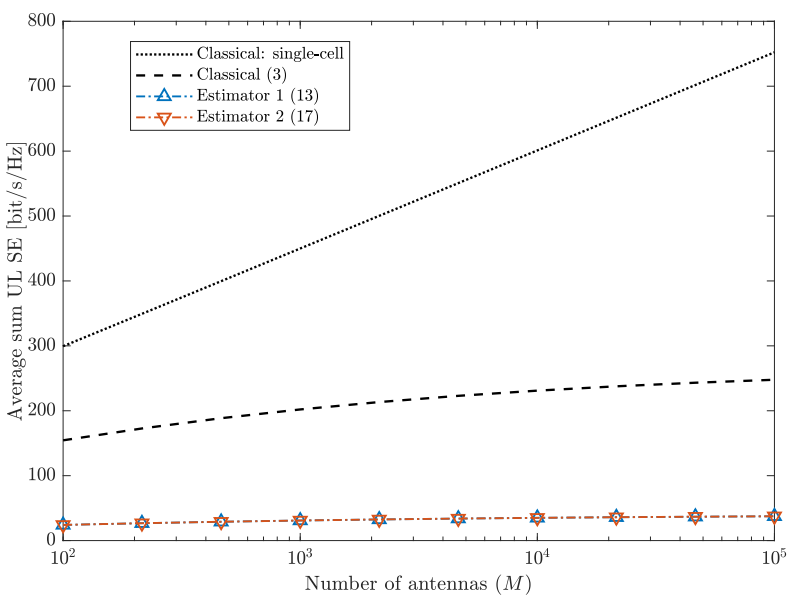

Source: The authors.

In spite of the fact that the average UL SE per UE is greater for the successive schemes than the classical one, the average sum UL SEs acquired by the evaluated techniques are much smaller than the classical case by virtue of $K_{\text {class. }} \gg K_{\text {succ. }}$. In fact, the average sum SEs supported by the successive cases are in average $84.72 \%$ smaller than the classical multi-cell scenario. This is a direct consequence of the excessive reduction of the spatial multiplexing gain for the successive approaches. Then, it can be inferred that a great fraction of the coherence interval is spent in the realization of multiple pilot training phases, which reduces the overall capacity of the system.

In addition to the decrease in the sum UL SE, the successive methods also need a complex synchronization across the cells, which embraces a challenging task to be performed. Moreover, we stress that if the total interval expended in the multiple phases, $L \tau_{\mathrm{p}}^{\text {succ. }}$, is applied to assign orthogonal pilot sequences to the $K_{\text {succ. UEs, the }}$ pilot contamination effect would be overcame without any complexity addition. In summary, the successive schemes do not secure a good trade-off between performance and complexity, turning out to be impracticable as $L$ increases.

\section{Conclusions}

In this work, the successive pilot decontamination method was deeply demonstrated, where such scheme relies on the coordination across the cells. To support our conclusions, the mathematical framework derived to represent the successive approach considered tight closed form expressions for the SE. In general, we concluded that the estimators proposed by VU; VU; QUEK (2014) are complicated from the implementation point of view, since they need the exact coordination of the communication phases of the cells. They also presented an enormous lack in the sum SE regarding the classical scheme. Therefore, it is possible to assert that multiple pilot training phases are not a good approach to overcome pilot contamination. In scenarios with a numerous quantity of cells, a simple pilot assignment with larger pilot sequences is sufficient to suppress the pilot contamination rather than the application of multiple pilot training phases.

\section{Acknowledgement}

This work was supported in part by Conselho Nacional de Desenvolvimento Científico e Tecnológico (CNPq) under Grant 102050/2018-0 and in part by Universidade Estadual de Londrina (UEL), under Grant PIBITI Scholarship 02/2018.

\section{References}

ELIJAH, O.; LEOW, C. Y.; RAHMAN, T. A.; NUNOO, S.; ILIYA, S. Z. A Comprehensive Survey of Pilot Contamination in Massive MIMO-5G System. IEEE Communications Surveys and Tutorials, 2016. New York, v. 18, n. 2, p. 905-923, 2016. ISSN 1553877X. 
HOYDIS, J.; BRINK, S. T.; DEBBAH, M. Massive MIMO in the UL/DL of cellular networks: How many antennas do we need? IEEE Journal on Selected Areas in Communications, 2013. New York, v. 31, n. 2, p. 160-171, 2013. ISSN 07338716.

KAY, S. M. Fundamentals of Statistical Signal Processing: Estimation Theory. [S.1.]: Prentice Hall, 1993. 242-251 p. ISSN 00401706. ISBN 0133457117.

MARZETTA, T. L.; LARSSON, E. G.; YANG, H.; NGO, H. Q. Fundamentals of Massive MIMO. Cambridge, United Kingdom: Cambridge University Press, 2016. 242 p. ISBN 9781316799895.

VU, T. X.; VU, T. A.; QUEK, T. Q. S. Successive pilot contamination elimination in multiantenna multicell networks. IEEE Wireless Communications Letters, 2014. New York, v. 3, n. 6, p. 617-620, 2014. ISSN 21622345. 